\title{
Energy Profile Prediction of Milling Processes Using Machine Learning Techniques
}

\author{
Matthias Mühlbauer, Hubert Würschinger, Dominik Polzer and Prof. Dr.-Ing. Nico \\ Hanenkamp \\ University, Erlangen-Nuremberg, Germany \\ Department of Mechanical Engineering \\ Institute of Resource and Energy Efficient Production Systems \\ matthias.muehlbauer@fau.de
}

\begin{abstract}
The prediction of the power consumption increases the transparency and the understanding of a cutting process, this delivers various potentials. Beside the planning and optimization of manufacturing processes, there are application areas in different kinds of deviation detection and condition monitoring. Due to the complicated stochastic processes during the cutting processes, analytical approaches quickly reach their limits. Since the 1980s, approaches for predicting the time or energy consumption use empirical models. Nevertheless, most of the existing models regard only static snapshots and are not able to picture the dynamic load fluctuations during the entire milling process. This paper describes a data-driven way for a more detailed prediction of the power consumption for a milling process using Machine Learning techniques. To increase the accuracy we used separate models and machine learning algorithms for different operations of the milling machine to predict the required time and energy. The merger of the individual models allows finally the accurate forecast of the load profile of the milling process for a specific machine tool. The following method introduces the whole pipeline from the data acquisition, over the preprocessing and the model building to the validation.
\end{abstract}

Keywords: energy prediction, time prediction, power load prediction, milling process, machine learning, regression

\section{$1 \quad$ Einleitung}

Der industrielle Sektor ist in Deutschland mit 28,9 \% am Gesamtenergieverbrauch (Stand: 2016, [1]) beteiligt, wobei ein wesentlicher Teil direkt auf zerspanende Prozesse zurückzuführen ist. Zunehmender politischer Druck, strengere Regulierungen und steigende Strompreise drängen die Hersteller zu immer nachhaltigeren, energieeffizienteren Produktionsprozessen. So führt ein Ausschussteil am Ende der Prozesskette einer spanenden Produktion zu einem Energieverlust von 60 bis 80 MJ pro Kilogramm Bauteilmasse [2]. Um schon vor Produktionsbeginn den Energiebedarf eines Bearbeitungsvorgangs, beispielsweise hinsichtlich der Reduktion von Lastspitzen, optimieren zu können, muss der Produktionsprozess transparent gemacht werden. Weiter trägt eine 
geschaffene Transparenz zur Identifikation von Anomalien im Bearbeitungsprozess bei, welche ggf. zu Ausschuss führen können. Beides ist über die Prognose des Energiebedarfs und dem folgenden Abgleich von Soll- und Ist-Werten bzw. der Optimierung von Parametereinstellungen möglich. Analytische Verfahren zur Prognose des Energiebedarfs, Ansätze, die auf den physikalischen Gesetzen der Fertigungsverfahren beruhen, stoßen im Bereich der Zerspanung schnell an Grenzen und werden heute kaum noch eingesetzt [3]. Diese Modelle haben das grundsätzliche Problem, dass sie die komplizierten stochastischen Prozessmechanismen während der Bearbeitung nicht ausreichend abbilden [4].

Eine weitere Möglichkeit ist die Modellierung des Energiebedarfs durch empirische Ansätze. Durch den vereinfachten Datenzugriff bei automatisierten Prozessen bieten Maschinelle Lernverfahren die Möglichkeit der Erstellung dynamischer Prognosemodelle. Erste Ansätze, die solche Verfahren für die Energieprognose verwenden, wurden in den vergangenen Jahren publiziert. Kant et al. nutzt Neuronale Netze zur Vorhersage des Energiebedarfs von einfachen Fräs- [5] als auch erster Drehprozesse [6]. In weiteren Veröffentlichungen nutzt der Autor u.a. klassische Techniken des Maschinellen Lernens wie die Support Vector Regression [7]. Während sich Kant in seinen Arbeiten auf die Datenaufnahme einfacher, statischer Prozesse fokussiert, betrachtet Park [8] unterschiedliche Fertigungsarten (wie z.B. Bohren und diverse Fräsverfahren) und nutzt dafür die Gaußprozess Regression.

Park und Kant konzentrieren sich in ihren Untersuchungen nur auf die Prognose des Energiebedarfs zerspanender Bearbeitungsschritte, abgeleitet aus Numerical Control (NC)-Datensätzen. Neben diesen Prognosemethoden des Energiebedarfs wurden noch Methoden zur Prognose des Zeit- und Wegbedarfs von Zerspanungsprozessen entwickelt. Hier lassen sich unterschiedliche Herangehensweisen unter Anwendung des Maschinellen Lernens identifizieren. Saric et al. [9] entwickelte in diesem Kontext ein Modell für die Zeitprognose, mit Hilfe Neuronaler Netze. Gopalakrishnan et al. [10] erläutert die Vorhersage der Werkzeugweglänge beim Taschenfräsen durch Neuronale Netze. Diese dient wiederum als Variable für die Abschätzung der Bearbeitungszeit und der entstehenden Kosten.

Die im Rahmen dieser Veröffentlichung dargestellte Methode geht einen Schritt weiter als bisher bekannte Ansätze und erhöht den Detailgrad der Prognose so, dass eine Abbildung des gesamten Funktionsumfangs der Bearbeitungsmaschine sowie der Einzelaktionen über die Zeit ermöglicht werden. Dies erfolgt durch getrennte Modelle, die die Prognose des Zeitbedarfs und des Energiebedarfs für verschiedene Aktionsschritte der Maschine repräsentieren. Dabei lassen sich einzelne Aktionen, wie z.B. die Drehzahländerungen der Spindel oder das Verfahren der Achsen, zu eigenständigen Prognosemodellen zusammenfassen. Über die Aggregation der Einzelmodelle lässt sich eine resultierende Leistungskurve erstellen, welche Aufschluss über die anliegende Last zu jedem Zeitpunkt gibt. Durch diese neue Methode kann ein detailliertes Abbild eines Zerspanprozesses auf Basis des Energiebedarfs über die Zeit erstellt werden, wodurch sich neue Möglichkeiten der Optimierung des Energiebedarfs sowie der Erkennung von Anomalien auf Ebene einzelner Maschinenaktionen ergeben. 


\section{Methode}

Anhand der folgenden Methode werden die Schritte von der Trainingsdatenerhebung über die Modellbildung sowie dessen Anwendung zur Prognose des Leistungsprofils beschrieben (vgl. Abb. 1). Die Vorgehensweise wird anhand einer Fräsbearbeitung aufgezeigt und validiert, jedoch ist die grundlegende Systematik auch auf weitere automatisierte Prozesse übertragbar. Der Datenvorverarbeitungs- und Modellbildungsvorgang erfolgt automatisiert und ermöglicht ein direktes Abgreifen der erforderlichen Daten während des Bearbeitungsprozesses. Die aufbereiteten Rohdaten ermöglichen im Weiteren die Erstellung von Regressionsmodellen, welche für die Prognose des Zeit- und Energiebedarfs genutzt werden. Abschließend wird das vollständige Leistungsprofil durch das Zusammensetzen aus Zeit- und Energiebedarf erstellt.

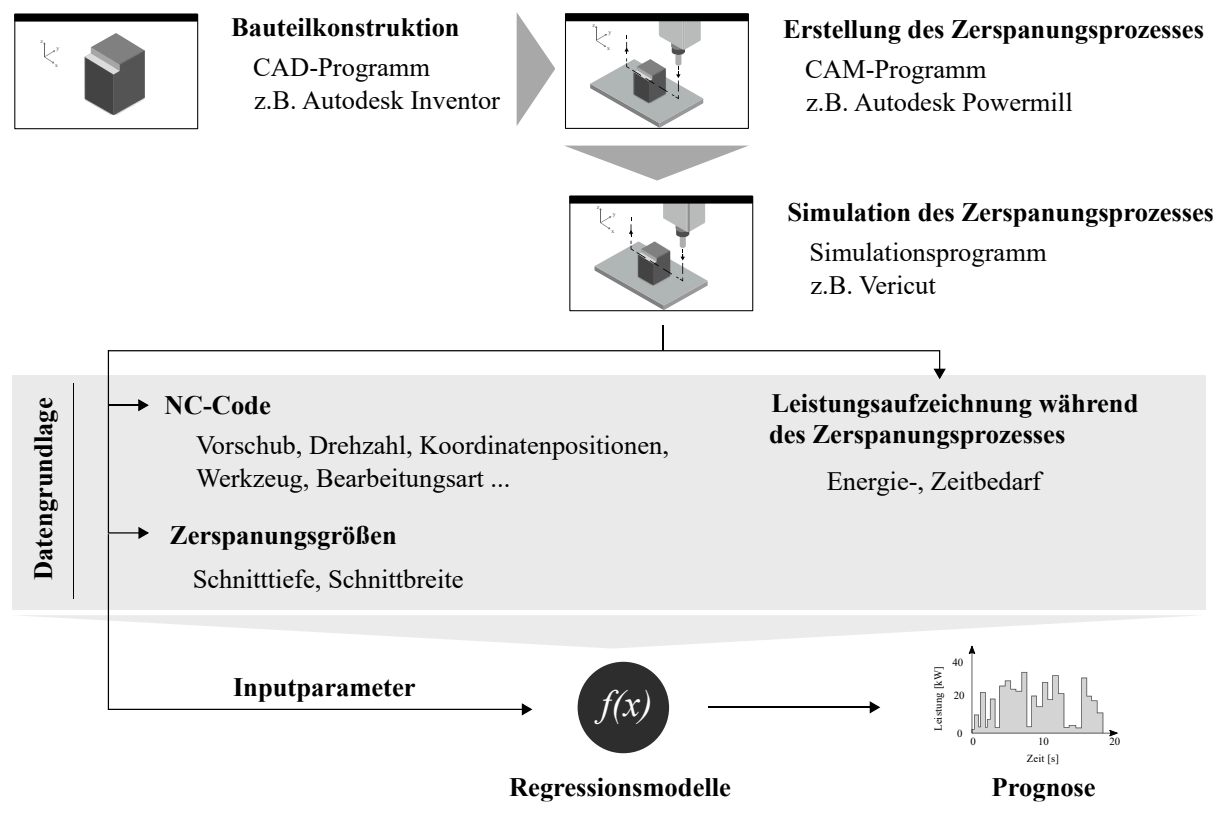

Abb. 1. Darstellung der entwickelten Methode

Der NC-Code beinhaltet bereits Informationen über Drehzahl, Vorschub, Verfahrweg und das verwendete Werkzeug. Für eine Prognose des Energiebedarfs, sowohl im Leerlauf als auch während des Zerspanvorgangs, sind weitere Daten über Schnittgrößen wie etwa Schnitttiefe und Schnittbreite während der Bearbeitung erforderlich. Diese werden mithilfe einer Simulation des Zerspanvorgangs ermittelt. Die relevanten Attribute, welche für die Durchführung der Modellbildung erforderlich sind, wurden im Vorfeld definiert.

Um das Leistungsprofil in der nötigen Güte prognostizieren zu können ist es erforderlich, die Prognose des Zerspanvorgangs in einzelne Elemente bzw. Einzelaktionen aufzuteilen und dabei die Maschinencharakteristik zu einer weiteren Erhöhung der 
Prognosegüte einzubeziehen. Sowohl bei Achsbewegung ohne Materialabtrag als auch bei Zerspanvorgängen selbst hat diese einen entscheidenden Einfluss auf den Zeitbedarf. Die Erstellung der Modelle auf Basis realer Datenaufzeichnungen der betrachteten Werkzeugmaschine ermöglicht eine genaue Abbildung dieser Einflussparameter.

\section{$3 \quad$ Datenerhebung und -aufbereitung}

Die Datenerhebung für die anschließende Modellbildung unterteilt sich in die Aufzeichnung der Zielwerte Energie- und Zeitbedarf und die Gewinnung der entsprechenden Werte der Einflussparameter. Als Datenquellen dienen hier NC-Befehlssätze, Simulation und Werkzeugmaschine. Um aufwendige Datenaufbereitungsvorgänge zu umgehen, werden für die Prognose des Leistungsverlaufs möglichst vorhandene Informationen aus den NC-Befehlssätzen genutzt. Fehlende Informationen zu Zerspanungsgrößen, wie etwa die Schnittbreite und Schnitttiefe während des Bearbeitungsvorganges, werden durch die Simulationssoftware Vericut berechnet und ausgeleitet. Dies ermöglicht eine Extraktion sämtlicher relevanter Inputparameter aus der Erstellung und Simulation des Zerspanungsvorgangs. Die Daten der Zielvariablen Energie- und Zeitbedarf werden durch Versuche an der Maschine aufgezeichnet und durch Schnittstellen extrahiert.

\subsection{Gewinnung der Zielwerte Energie- und Zeitbedarf}

Das im Rahmen dieser Arbeit betrachtete Bearbeitungszentrum (Doosan DNM 500 II) ermöglicht die Aufzeichnung der Leistungsaufnahme der einzelnen Verbraucher über eine Siemens Numerik Steuerung (Sinumerik 828D). Diese Leistungsaufzeichnungen dienen als Grundlage für die nachfolgende Modellbildung. Um die erforderliche Datenbasis für die Modellbildung zu schaffen, wurden bei insgesamt 32 Planfräsprozessen, 186 Verfahrvorgänge der Achsen und 30 Drehzahländerungsvorgänge die Wirkleistungsverläufe der wesentlichen Verbraucher, der drei Achsen und der Spindel, aufgezeichnet. Es wurde eine Routine entwickelt, um die Daten automatisiert aufzubereiten und vorzuverarbeiten. Während der Aufbereitung der Daten werden die Energieund Zeitbedarfe der einzelnen Bearbeitungsschritte berechnet und die Werte der Eingangs- und Zielvariablen in den entsprechenden Datensätzen für das Anlernen der einzelnen Modelle abgespeichert.

\subsection{Gewinnung der Inputparameter für die Regressionsmodelle}

Um den Energiebedarf auch bei einem Werkzeugeingriff ausreichend genau abbilden zu können, sind neben den Daten aus den NC-Befehlen Informationen zu den Spanungsgrößen erforderlich. Für die Gewinnung der für die Prognose relevanten Daten wird die Simulationssoftware Vericut verwendet. Diese ermöglicht eine Simulation der Werkzeugwege und der Materialabtragsprozesse. Die hierbei berechneten Spanungsgrößen können zusammen mit den NC-Datensätzen aus dem Programm ausgeleitet werden und bilden die Basis für die Erstellung der Regressionsmodelle. Für die 
anschließende Prognose des Leistungsverlaufs eines betrachteten Zerspanungsprozesses wird ebenso verfahren. Die Zerspanungsprozesse werden in der Regel mit Hilfe einer CAM-Software simuliert. Eine zusätzliche Überführung des Bearbeitungsprogrammes in Vericut liefert weitere Inputparameter, welche für die Energie- und Zeitprognose erforderlich sind.

\subsection{Feature Engineering}

Im Falle der Energieprognose gilt es, über Feature Engineering, die Einflussparameter zu identifizieren, die Auswirkung auf den Energiebedarf haben und in ihrer Gesamtheit somit eine möglichst genaue Prognose ermöglichen. Anhaltspunkte hierfür geben die Literatur, Domänenwissen und die bereits bestehenden Modelle zur Berechnung der Schnittkraft. Kühn et al. [11] nennt zehn Einflussgrößen, die sich auf die Schnittkraft $F_{C}$ und somit direkt auf die benötigte Leistung auswirken: Werkstoff, Vorschub bzw. Spanungsdicke, Schnitttiefe bzw. Spanungsbreite, Spanungsverhältnis (Schnitttiefe/Vorschub), Spanwinkel, Einstellwinkel, Schnittgeschwindigkeit, Schneidstoff, Kühlung und Schmierung und Werkzeugverschleiß.

\section{$4 \quad$ Modellbildung}

Da mit dieser Methode der Energiebedarf des gesamten Funktionsumfanges der Bearbeitungsmaschine, repräsentiert durch unterschiedliche Verbraucher, abgedeckt werden soll und sich die Einflussparameter auf den Energiebedarf je nach Aktionsschritt stark unterscheiden, erfolgt die Modellbildung auf Einzelaktionsebene, siehe Abb. 2.

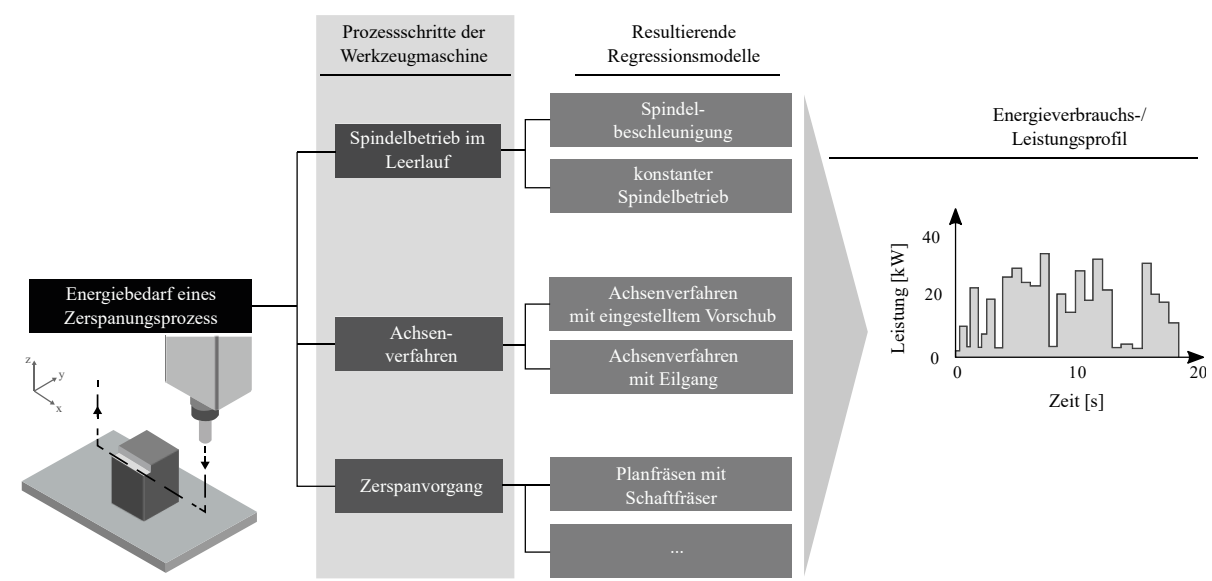

Abb. 2. Regressionsmodelle der Einzelaktionen des Bearbeitungsprozesses

Zum einen werden verschiedene Modelle für zeitgleich aktive Verbraucher, wie z. B. Spindelantrieb und Achsantriebe, erstellt. Zum anderen werden auch für dieselben Verbraucher verschiedene Modelle erstellt, wenn diese stark unterschiedliche Aktionen durchführen, wie z. B. das Verfahren der Achsen im Normalbetrieb bzw. im Eilgang. 
Die Aufteilung in verschiedene Einzelaktionen bzw. Modelle erfolgte mit der Prämisse der Maximierung der Leistungsfähigkeit der Einzelmodelle und somit der Prognosegenauigkeit unter der Berücksichtigung einer noch vertretbaren Anzahl sich ergebender Modelle. Die erstellten Einzelmodelle werden abschließend in einem Gesamtmodell aggregiert, welches die Prognose des Leistungsprofils ermöglicht.

Konnte eine definierte Einzelaktion nicht ausreichend durch ein Modell abgebildet werden, erfolgte eine detailliertere Untersuchung der zugrundeliegenden Datenstruktur. Beispielweise wurde beim Zeitbedarf für das Verfahren der Achsen im Eilgang eine Knickstelle bei der Analyse der Daten festgestellt. Durch diese spezielle Datenstruktur ist eine separate Modellbildung links und rechts der Knickstelle sinnvoll, siehe Abb. 3. Weder die Gaußprozess Regression noch die Polynomiale Regression können die vollständigen Daten richtig annähern. Durch die Aufteilung in zwei Modelle gelingt eine verbesserte Annäherung.

Gauß-Prozess Regression $\mathrm{R} 2=0,652$

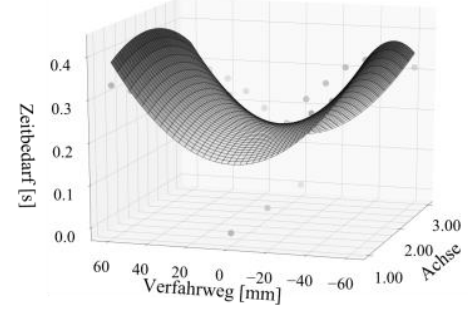

Aufteilung in zwei Teilmodelle

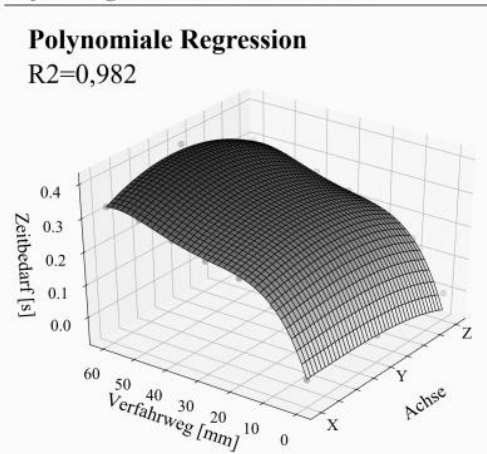

\section{Polynomiale Regression} $\mathrm{R} 2=0,723$

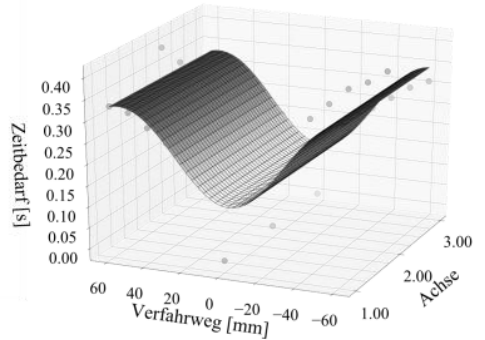

Abb. 3. Verbesserung der Prognosegüte durch Modellierung von zwei Teilmodellen

Während des Modellbildungsprozesses wurden für die einzelnen Datensätze der definierten Aktionen unterschiedliche Regressionsverfahren mit Hilfe der Kreuzvalidierung (Leave-One-Out) evaluiert. Betrachtet wurde dabei die Leistungsfähigkeit der Linearen, Polynomialen, Gaußprozess und Random Forest Regression über die Evaluati- 
onsmetrik Mittlere Quadratische Abweichung (MSE) und die Mittlere Absolute Abweichung (MAE). Über eine Routine konnten die Hyperparameter der Verfahren automatisch variiert und bewertet werden. Das Gütemaß MSE diente hierbei als Optimierungsgröße. In Tabelle 1 werden die Unterschiede der Regressionsverfahren am Beispiel der Aktion Drehzahländerung verdeutlicht. Je Einzelaktion wurde das Regressionsverfahren bestimmt, welches die geringste Abweichung (MSE) aufweist und somit die jeweilige Aktion am besten annähert. Das finale Gesamtmodell zur Prognose des Leistungsverlaufs setzt sich dementsprechend aus den elf gewählten Teilmodellen zusammen.

Tab. 1. Vergleich der Regressionsverfahren zur Prognose des Energiebedarfs der Aktion Drehzahländerung

\begin{tabular}{llll}
\hline Regressionsverfahren & Hyperparameter & MAE $[\mathbf{k J}]$ & MSE $\left[\mathbf{k J} \mathbf{2}^{2}\right]$ \\
\hline Lineare Regression & & 3,21 & 13,30 \\
Polynomiale Regression & Polynomgrad=2 & 0,55 & 0,69 \\
Random Forest & Bäume=10; max. Tiefe $=10$ & 2,62 & 9,29 \\
Gaußprozess Regression & Kernel = RBF & 0,81 & 1,05 \\
\hline
\end{tabular}

\section{$5 \quad$ Ergebnisse und Validierung}

Sämtliche Regressionsmodelle sind speziell auf die betrachtete Bearbeitungsmaschine abgestimmt und bilden somit Maschinenspezifika mit ab. Dies ermöglicht hohe Prognosegenauigkeiten.

Tab. 2. Teilregressionsmodelle für den Energiebedarf von Zerspanungsprozessen

\begin{tabular}{|c|c|c|c|c|c|}
\hline $\begin{array}{l}\text { Regressi- } \\
\text { onsverfah- } \\
\text { ren }\end{array}$ & Hyperparameter & Attribute & $\mathbf{R}^{2}$ & $\begin{array}{l}\text { MSE } \\
{\left[\mathbf{k J}^{2}\right]}\end{array}$ & \\
\hline \multicolumn{6}{|c|}{ Energiemodell: Achsenverfahren im Eilgang } \\
\hline $\begin{array}{l}\text { Polynomiale } \\
\text { Regression }\end{array}$ & Polynomgrad $=4$ & $\begin{array}{l}\text { Verfahrweg (und Richtung) } \\
{[\mathrm{mm}], \text { Achse }[\mathrm{x}=1, \mathrm{y}=2, \mathrm{z}=3]}\end{array}$ & 0,986 & $\begin{array}{l}2,07 \\
10^{-4}\end{array}$ & \\
\hline \multicolumn{6}{|c|}{ Energiemodell: Achsenverfahren mit eingestelltem Vorschub } \\
\hline $\begin{array}{l}\text { Polynomiale } \\
\text { Regression }\end{array}$ & Polynomgrad $=4$ & $\begin{array}{l}\text { Verfahrweg (und Richtung) } \\
{[\mathrm{mm}], \text { Achse }[x=1, y=2, \mathrm{z}=3] \text {, }} \\
\text { Vorschub } \mathrm{f}[\mathrm{mm} / \mathrm{min}]\end{array}$ & 0,982 & $\begin{array}{l}1,72 \\
10^{-4}\end{array}$ & \\
\hline \multicolumn{6}{|c|}{ Energiemodell: Drehzahländerung } \\
\hline $\begin{array}{l}\text { Polynomiale } \\
\text { Regression }\end{array}$ & Polynomgrad $=2$ & $\begin{array}{l}\text { Ausgangsdrehzahl [1/min], End- } \\
\text { drehzahl [1/min] }\end{array}$ & 0,992 & 0,26 & \\
\hline \multicolumn{6}{|c|}{ Energiemodell: Werkzeug (Schaftfräser) im Eingriff } \\
\hline $\begin{array}{l}\text { Polynomiale } \\
\text { Regression }\end{array}$ & Polynomgrad $=2$ & $\begin{array}{l}\text { Verfahrweg }[\mathrm{mm}], \text { Vorschub } \\
{[\mathrm{mm} / \mathrm{min}], \text { Drehzahl }[1 / \mathrm{min}]} \\
\text { Schnitttiefe }[\mathrm{mm}], \text { Schnittbreite } \\
{[\mathrm{mm}]}\end{array}$ & 0,980 & 0,22 & \\
\hline \multicolumn{6}{|c|}{ Energiemodell: konstanter Spindelbetrieb } \\
\hline $\begin{array}{l}\text { Random Fo- } \\
\text { rest }\end{array}$ & $\begin{array}{l}\text { Bäume }=10 ; \\
\text { Max. Tiefe }=20\end{array}$ & Drehzahl n [1/min] & 0,604 & 0,11 & \\
\hline
\end{tabular}


Die weitere Evaluation der Modelle erfolgte auf Basis eines Testdatensatzes. Tabelle 2 zeigt die für die Energieprognose verwendeten Regressionsverfahren, die relevanten Hyperparametereinstellungen, die Merkmale der Modelle und die Evaluationsergebnisse anhand des Bestimmtheitsmaßes $\left(\mathrm{R}^{2}\right)$ und der mittleren quadratischen Abweichung (MSE).

Das Bestimmtheitsmaß nimmt bei den meisten Modellen einen Wert über 0,9 ein. Die Ausnahme bildet hier das Modell für die Prognose des Energiebedarfs bei konstantem Spindelbetrieb. Es wird angenommen, dass der verwendete Datensatz mit lediglich sieben Instanzen ursächlich für die geringere Modellgüte ist. Die Ergebnisse der mittleren quadratischen Abweichungen (MSE) der Prognosewerte spiegeln diese Ergebnisse wieder.

Hiermit konnte gezeigt werden, dass eine Prognose des Energiebedarfs auf Einzelaktionsebene durch die gewählten Verfahren und Attribute mit hoher Genauigkeit möglich ist. Gleichwertige Ergebnisse konnten für die Prognose des Zeitbedarfs erzielt werden (vgl. Tabelle 3). Die Modelle für den Zeitbedarf erreichen durchgehend ein Bestimmtheitsmaß über 0,9 .

Tab. 3. Teilregressionsmodelle für den Zeitbedarf von Zerspanungsprozessen

\begin{tabular}{|c|c|c|c|c|}
\hline $\begin{array}{l}\text { Regressi- } \\
\text { onsverfah- } \\
\text { ren }\end{array}$ & Hyperparameter & Attribute & $\mathbf{R}^{2}$ & $\begin{array}{l}\text { MSE } \\
{\left[\mathbf{s}^{2}\right]}\end{array}$ \\
\hline \multicolumn{5}{|c|}{ Zeitmodell: Achsenverfahren im Eilgang (in positive Koordinatenrichtung) } \\
\hline $\begin{array}{l}\text { Polynomiale } \\
\text { Regression }\end{array}$ & Polynomgrad $=4$ & $\begin{array}{l}\text { Verfahrweg (und Richtung) [mm }] \\
\text { Achse }[x=1, y=2, z=3]\end{array}$ & 0,935 & $\begin{array}{l}1,16^{*} \\
10^{-3}\end{array}$ \\
\hline \multicolumn{5}{|c|}{ Zeitmodell: Achsenverfahren im Eilgang (in negative Koordinatenrichtung) } \\
\hline $\begin{array}{l}\text { Polynomiale } \\
\text { Regression }\end{array}$ & Polynomgrad $=4$ & $\begin{array}{l}\text { Verfahrweg (und Richtung) }[\mathrm{mm}] \text {, } \\
\text { Achse }[x=1, y=2, z=3]\end{array}$ & 0,982 & $\begin{array}{l}3,19 * \\
10^{-4}\end{array}$ \\
\hline \multicolumn{5}{|c|}{ Zeitmodell: Achsenverfahren mit eingestelltem Vorschub } \\
\hline $\begin{array}{l}\text { Polynomiale } \\
\text { Regression }\end{array}$ & $\begin{array}{l}\text { Bäume }=20 \\
\text { Max. Tiefe }=20\end{array}$ & $\begin{array}{l}\text { Verfahrweg (und Richtung) }[\mathrm{mm}] \text {, } \\
\text { Achse }[\mathrm{x}=1, \mathrm{y}=2, \mathrm{z}=3] \text {, Vorschub } \\
\mathrm{f}[\mathrm{mm} / \mathrm{min}]\end{array}$ & 0,994 & $\begin{array}{l}1,85 * \\
10^{-2}\end{array}$ \\
\hline \multicolumn{5}{|c|}{ Zeitmodell: Drehzahländerung } \\
\hline $\begin{array}{l}\text { Gauß-Pro- } \\
\text { zess } \\
\text { Regression }\end{array}$ & $\begin{array}{l}\text { Rational Quadratic } \\
\text { Kernel; Alpha }=0,1 ; \\
\text { Varianz }=1,0 ; \\
\text { Lenghtscale }=1,0\end{array}$ & $\begin{array}{l}\text { Ausgangsdrehzahl }[1 / \mathrm{min}] \text {, End- } \\
\text { drehzahl }[1 / \mathrm{min}]\end{array}$ & 0,942 & $\begin{array}{l}6,37 * \\
10^{-3}\end{array}$ \\
\hline
\end{tabular}

Die Prognosewerte werden im Weiteren zur Berechnung und Darstellung des gesamten Leistungsverlaufs genutzt. Auf Basis der prognostizierten Zeitbedarfe für die Einzelaktionen und der Berücksichtigung, ob diese parallel bzw. seriell stattfinden, ergibt sich die geplante Dauer der Einzelaktion und des gesamten Bearbeitungsvorgangs. Die prognostizierten Energiebedarfe werden den entsprechenden Zeitbedarfen zugeordnet, wodurch der Leistungsverlauf ausgeprägt wird und folgend die Ermittlung des Gesamtenergiebedarfs durch Integration ermöglicht. Abb. 4 stellt die reale Leistungsaufzeichnung während eines Planfräsprozesses und die der Prognose gegenüber. Die prognostizierte Leistungskurve spiegelt den realen Verlauf erkennbar wider. Weiter decken sich die prognostizierten und realen Energiebedarfswerte für die definierten Aktionen. Die 
absolute Abweichung (MAE) des Beispielprozesses eines Planfräsvorgangs beträgt $1,02 \mathrm{~kJ}$, was einer Abweichung von unter $4 \%$ entspricht.

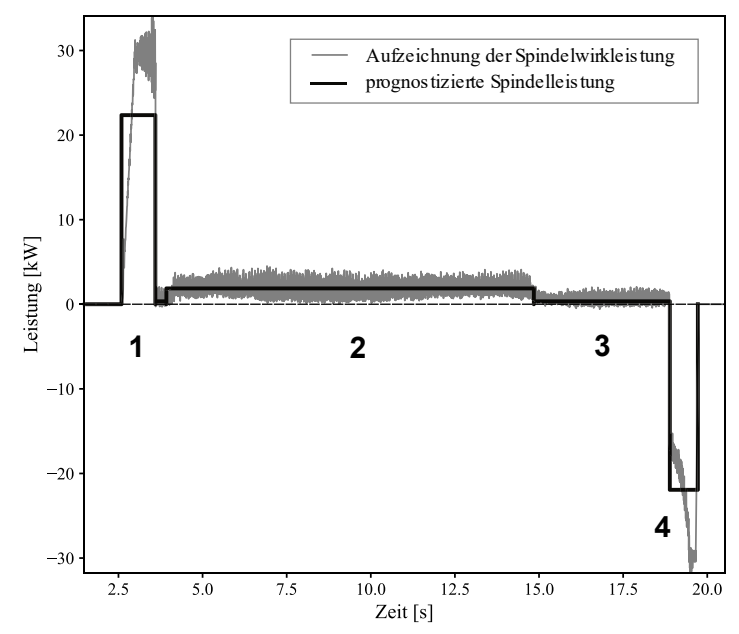

$$
\begin{aligned}
& \text { 1. Spindelbeschleunigung } \\
& \mathrm{W}_{\mathrm{i} \mathrm{st}_{\mathrm{t}}}=23,23 \mathrm{~kJ} \\
& \mathrm{~W}_{\mathrm{p}_{\mathrm{r}} \mathrm{g}_{\mathrm{n}} \mathrm{o}_{\mathrm{s}} \mathrm{e}}=22.86 \mathrm{~kJ}
\end{aligned}
$$

2.Planfräsvorgang

$\mathrm{W}_{\mathrm{is}_{\mathrm{t}}}=21,24 \mathrm{~kJ}$

$\mathrm{W}_{\mathrm{pr}_{\mathrm{O}} \mathrm{g}_{\mathrm{n}} \mathrm{S} \mathrm{e}}=20,98 \mathrm{~kJ}$

3. Verfahrbewegung der X-Achse

$\mathrm{W}_{\mathrm{ist}_{\mathrm{t}}}=0,058 \mathrm{~kJ}$

$\mathrm{W}_{\mathrm{pr}_{\mathrm{O}} \mathrm{g}_{\mathrm{n}} \mathrm{O}_{\mathrm{s}} \mathrm{e}}=0,069 \mathrm{~kJ}$

4. Spindelabbremsung

$\mathrm{W}_{\mathrm{is}_{\mathrm{t}}}=-18,83 \mathrm{~kJ}$

$\mathrm{W}_{\mathrm{p}^{\mathrm{r}} \mathrm{g} \mathrm{g}_{\mathrm{s}} \mathrm{e}}=-19,23 \mathrm{~kJ}$

Abb. 4. Vergleich der Prognose und des aufgezeichneten Leistungsverlaufs während eines Zerspanungsprozesses (Beispiel: Planfräsvorgang mit einem $12 \mathrm{~mm}$ Schaftfräser, Drehzahl: 7000 1/min, Vorschub: $560 \mathrm{~mm} / \mathrm{min}$, Schnitttiefe: $6 \mathrm{~mm}$, Schnittbreite: $9 \mathrm{~mm}$ )

\section{Diskussion und Ausblick}

Die vorgestellte Methode demonstriert die Prognose des Leistungsprofils bzw. des Energiebedarfs eines Bearbeitungszentrums mit hoher Genauigkeit auf Einzelaktionsebene. Hierdurch ergeben sich Potentiale im Hinblick auf diverse Optimierungsmaßnahmen. Diese ergeben sich durch die Optimierung von Prozessparametern hinsichtlich der Minimierung des Zeit- oder Energiebedarfs des Zerspanvorgangs, dem Mikro-Lastmanagement bzw. der Vermeidung von Lastspitzen bei der Berücksichtigung von mehreren parallellaufenden Prozessen und auch im Bereich der Anomaliedetektion durch einen Vergleich von Soll- und Ist-Werten.

Obwohl die beschriebene Methode schon weitestgehend automatisiert wurde, ist eine weitere Automatisierung der Vorgehensweise anzustreben. Insbesondere die Erhebung von maschinenspezifischen Trainingsdaten gestaltet sich noch aufwändig. Hier ist ein einfach implementierbares Edge-Device mit den erforderlichen Schnittstellen zur Datenerhebung an der Maschine und mit der Möglichkeit zur Datenvorverarbeitung denkbar. Um die genannten Potentiale zur Anomalieerkennung ausschöpfen zu können, ist zudem ein Abgleich von Soll- und Ist-Werten in Echtzeit erforderlich. 


\section{References}

1. Umweltbundesamt auf Basis Arbeitsgemeinschaft Energiebilanzen: Endenergieverbrauch 2017 nach Sektoren. Auswertungstabellen zur Energiebilanz für die Bundesrepublik Deutschland 1990 bis 2017, Stand 07/2018

2. Fraunhofer Gesellschaft, Energieeffizienz in der Produktion: Untersuchung zum Handlungsund Forschungsbedarf. München (2008)

3. Bhinge, R.; Park, J.; Law, K. H.; Dornfeld, D. A.; Helu, M.; Rachuri, S.: Toward a Generalized Energy Prediction Model of Machine Tools. In: Journal of Manufacturing Science and Engineering (2017)

4. Duerden, C.; Shark, L.-K.; Hall, G; Howe, J.: Prediction of Granular Time-Series Energy Consumption for Manufacturing Jobs from Analysis and Learning of Historical Data. In: 2016 Annual Conference on Information Science and Systems (2016)

5. Kant, G.; Sangwan, K. S.: Predictive Modelling for Energy Consumption in Machining Using Artificial Neural Network. In: Procedia CIRP (2015)

6. Sangwan, K. S.; Kant, G.: Optimization of Machining Parameters for Improving Energy Efficiency using Integrated Response Surface Methodology and Genetic Algorithm Approach. In: Procedia CIRP (2017)

7. Kant, G.: Prediction and Optimization of Machining Parameters for Minimizing Surface Roughness and Power Consumption during Turning of AISI 1045 Steel. Birla Institute of Technology \& Science, Pilani (2016)

8. Park, J.; Law, K. H.; Bhinge, R.; Biswas, N.: A Generalized Data-Driven Energy Prediction Model with Uncertainty for a Milling Machine Tool Using Gaussian Process. In: Volume 2: Materials; Biomanufacturing; Properties, Applications and Systems; Sustainable Manufacturing (2015)

9. Saric, T.; Simunovic, G.; Simunovic, K.: Estimation of Machining Time for CNC Manufacturing Using Neural Computing. In: International Journal of Simulation Modelling (2016)

10. Gopalakrishnan, B.; Reddy, V. K.; Gupta, D. P.: Neural networks for estimating the tool path length in concurrent engineering applications. In: Journal of Intelligent Manufacturing (2004)

11. Kühn, K.-D.; Fritz, A. H.; Förster, R.; Hoffmeister, H.-W.: Trennen. In: Fertigungstechnik. 12. Auflage, Hrsg. Fritz, A. H., Springer Vieweg, Berlin (2018) 
Open Access This chapter is licensed under the terms of the Creative Commons Attribution 4.0 International License (http://creativecommons.org/licenses/by/4.0/), which permits use, sharing, adaptation, distribution and reproduction in any medium or format, as long as you give appropriate credit to the original author(s) and the source, provide a link to the Creative Commons license and indicate if changes were made.

The images or other third party material in this chapter are included in the chapter's Creative Commons license, unless indicated otherwise in a credit line to the material. If material is not included in the chapter's Creative Commons license and your intended use is not permitted by statutory regulation or exceeds the permitted use, you will need to obtain permission directly from the copyright holder. 